\title{
Indoor Environmental Monitoring System Using a Robot Vacuum Cleaner
}

\author{
Kazutoshi Noda* and Hidenobu Aizawa \\ Environmental Management Research Institute, \\ National Institute of Advanced Industrial Science and Technology (AIST), \\ 16-1 Onogawa, Tsukuba, Ibaraki 305-8569, Japan \\ (Received April 24, 2019; accepted January 16, 2020)
}

Keywords: IoT, robot, quartz crystal microbalance, semiconductor gas sensor, environmental measurement, automatic operation

We have studied a method of monitoring an indoor environment using an advanced robot vacuum cleaner equipped with a small sensor unit consisting of temperature, humidity, and gas sensors. We conducted a test to detect a vaporized material on the floor in a room. The sensor unit (temperature, humidity, air pressure, quartz crystal microbalance, and semiconductor gas sensors) was used in the test. Measurements were made with the sensor unit placed in the dust box of the vacuum cleaner. To represent a leaked liquid, a given number of water droplets and a sponge soaked with a given volume of ethanol were placed at different locations on the floor, and we investigated the detection characteristics of each sensor when the cleaner passed by the droplets and sponge representing the leaked liquid. In all the detection tests, measurements made with the sensor unit placed in the dust box showed the selective detection of water and ethanol. The most likely reason for the increase in detection output is that a few water droplets on the floor were distributed by the brush and vaporized to inside the robot vacuum cleaner, where they were detected by the sensor unit in the dust box. We showed that this form of monitoring has the potential to detect a spilled or leaked liquid.

\section{Introduction}

Internet of Things (IoT) technology connects various objects to the Internet and is bringing significant changes to our living environment. A typical example is the development and rapid spread of communications devices such as smartphones. Various sensor devices that are becoming ever smaller and more multifunctional are built into smartphones. The number of homes with an AI speaker is increasing. With an AI speaker or smartphone, you can remotely turn on the lights, air-conditioning unit, TV, robot vacuum cleaner, and other appliances.

To live a safe and secure life, it is important to be able to monitor indoor environments in real time. ${ }^{(1-6)}$ Indoor environments, such as indoor facilities, are not sealed spaces. For example, some homes are equipped with a $24 \mathrm{~h}$ ventilation system. The leakage of a flammable or explosive gas, such as city gas, can be hazardous even in ventilated indoor environments.

*Corresponding author: e-mail: kazu-noda@aist.go.jp

https://doi.org/10.18494/SAM.2020.2413 
This is true not only for domestic spaces but also for facilities such as manufacturing plants. In indoor spaces with a high risk of gas leakage, gas sensors need to be installed in multiple locations to ensure $24 \mathrm{~h}$ monitoring. However, it is not realistic to install multiple sensors in places that have a low risk of gas leakage, such as homes. There are potential dangers in homes, such as water leakage due to human error or pipe degradation. The frequency of gas and water leakage is very low, but the risk remains.

Ideally, it is desirable to monitor the indoor environment with autonomous devices, such as robots. Research in this area is being conducted. ${ }^{(7-23)}$ A robot should detect anomalous areas: a self-propelled type is an ideal means of performing this task. However, owing to the many issues remaining to be resolved, such robots are not yet being practically applied.

We have studied a method of monitoring an indoor environment using an advanced robot vacuum cleaner equipped with a small sensor unit consisting of temperature, humidity, and gas sensors, although it may not be as ideal as a search robot.

Robot vacuum cleaners can clean the corners of a room very efficiently and are available at affordable prices. The number of households using robot vacuum cleaners is increasing. However, sensors to detect anomalies are not mounted on such cleaners. We placed a sensor unit in the dust box of a vacuum cleaner. The outputs from the sensors are wirelessly transmitted to an external device, such as a PC, for the purpose of monitoring. We define the use of a robot such as this for the simple scanning of an entire environment, regardless of whether or not there are anomalous areas, as passive monitoring.

We here study a passive indoor environment monitoring method using a commercially available robot vacuum cleaner and a small sensor unit.

\section{Materials and Methods}

We conducted a test to detect a vaporized material originating from a given number of water droplets and a sponge soaked with a given amount of ethanol placed at different locations on the floor in a room. Since the robot vacuum cleaner we employed is of the ordinary domestic type, unlike the active search robots currently being developed, it cannot autonomously move toward the leaked liquid. Domestic robot vacuum cleaners have several operating modes. A mode for quickly cleaning a relatively wide area was used in the test.

The sensor unit we used is described below (Fig. 1). The sensors in the unit are commercially available temperature/humidity sensors (Sensirion; SHT15) and an air pressure sensor (Bosch; BMP180). The unit can measure the frequency of a quartz crystal microbalance (QCM). A QCM sensor with Ag electrodes [AT-cut, fundamental frequency $\left(f_{0}\right)=12 \mathrm{MHz}$ ] was used. Typically, a QCM sensor with an organic thin film formed on the electrode is significantly affected by gases and moisture, and a QCM sensor without an organic thin film on the electrode can be used to detect moisture. A commercially available semiconductor gas sensor (Figaro; TGS8100) was used in addition to the QCM sensor. This gas sensor is particularly highly sensitive to combustible gases such as hydrogen. It is also sensitive to alcohol, isobutane, and carbon monoxide as it is designed to detect contaminated air. The QCM and semiconductor gas sensors were used in the test. The sensor outputs can be viewed on an external PC in real time 


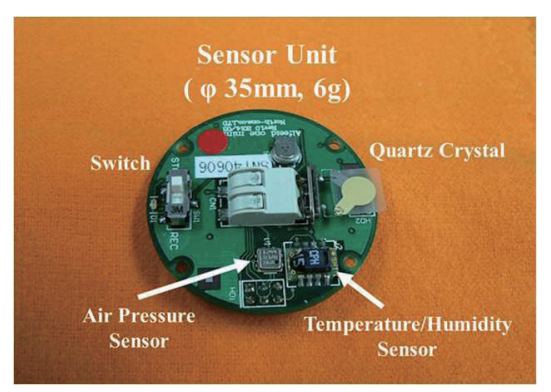

\begin{tabular}{lc}
\hline QCM $(\mathrm{MHz})$ & $6-30$ \\
\hline Temperature $\left({ }^{\circ} \mathrm{C}\right)$ & $-40-123$ \\
Humidity $(\% \mathrm{RH})$ & $0-100$ \\
Air pressure $(\mathrm{hPa})$ & $300-1100$ \\
\hline & DC 3 V \\
Power supply & Internal memory, \\
Function & 35 mm dia., \\
& about 6 g \\
\hline
\end{tabular}

Fig. 1. (Color online) Specifications of the sensor unit.

by mounting a wireless transmission board on the sensor unit. The detection data received from the sensors can also be saved in the internal memory of the sensor unit.

In the test, measurements were made with the sensor unit placed in the cleaner's dust box. With this configuration, air (gas) near the floor's surface is drawn in and brought into contact with the sensors, thus improving the response of the sensor. We measured the indoor environment using this setup. In addition to real-time monitoring with wireless data transmission, we used a method in which the data saved in the memory of the small sensor unit could be retrieved and analyzed after the end of the test.

The robot vacuum cleaner used in the test was a HOM-BOT Square (VR6270LVMB; LG Electronics) (Fig. 2). In the test, measurements were made with the sensor unit placed in the cleaner's dust box (about $170 \times 55 \times 40 \mathrm{~mm}^{3}$ ) [Fig. 2(c)]. In each experiment, the sensor unit was placed in the area shown in the figure to ensure a similar condition. The sensor unit was placed in a filter to prevent contamination with dust and particles. This cleaner has a built-in camera on the top and its location can be detected using travel information from the encoder and images of objects above the cleaner, enabling accurate autonomous travel using simultaneous localization and mapping (SLAM).

The test was conducted in a laboratory (Fig. 3) at the National Institute of Advanced Industrial Science and Technology. The cleaner moved around a room measuring approximately $7.13 \times 6.3 \mathrm{~m}^{2}$ with a laboratory bench in the center with dimensions of about $2.3 \times 1.5 \mathrm{~m}^{2}$. This test is similar to cleaning a room. Therefore, the amount of dust in the room decreases as the test proceeds. Since the original uniformly dusty surface of the floor could not be reproduced in subsequent tests, the tests were repeated under an "as-is" condition. The time taken to sweep the area was about $17 \pm 2 \mathrm{~min}$. To represent a leaked liquid, a given number of water (pure water) droplets and a sponge soaked with a given amount of ethanol (FUJIFILM Wako Pure Chemical Co., 95\%, Guaranteed Reagent) were placed at different locations on the floor. We planned to conduct the test using a gaseous liquid heavier than air but did not implement it owing to the difficulty in keeping the liquid in the same location without it diffusing away.

We investigated the detection characteristics of each sensor when the cleaner passed by the droplets representing a trace of leaked liquid. 


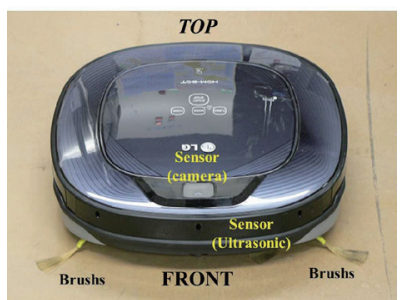

(a)

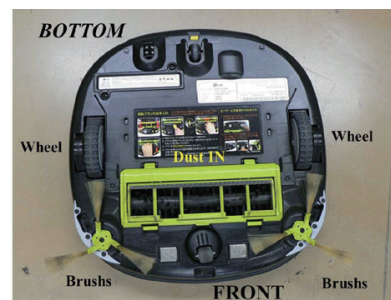

(b)

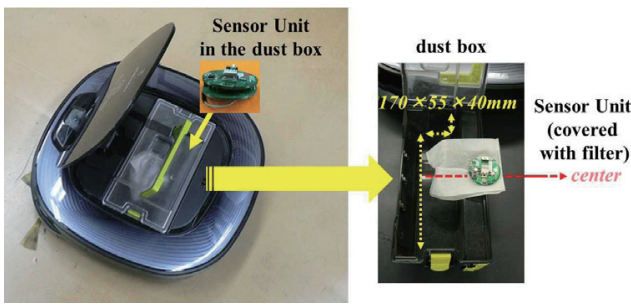

(c)

Fig. 2. (Color online) Robot vacuum cleaner used in test. (a) External view (top), (b) air inlet (bottom), and (c) installation of the sensor unit.

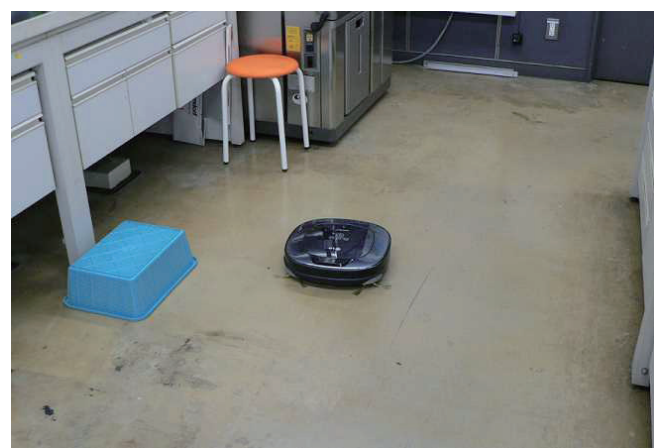

Fig. 3. (Color online) Example of testing by a robot vacuum cleaner.

\section{Results and Discussion}

In the indoor test, measurements were made using the sensors under the following conditions.

1) The original floor surface (without water or ethanol droplets on it) was not modified in any way.

2) $0.5 \mathrm{~mL}$ of water was dropped onto the floor surface at one location and a sponge soaked with $10 \mathrm{~mL}$ of ethanol was placed at another location.

Figure 4 shows an example of the measurement data, and Fig. 5 shows the traveling route of the robot cleaner and the location of the liquid. In all the detection tests, the measurements made with the sensor unit placed in the dust box showed the selective detection of water and ethanol, although the conditions were not exactly the same in all the tests.

The suction power of the LG robot vacuum cleaner, measured as the pressure difference between the air inlet and the air outlet, was about $0.2-0.4 \mathrm{hPa}$, according to the output of the air pressure sensor. The air speed near the air outlet was about $0.2-0.6 \mathrm{~m} / \mathrm{s}$. The air flow rate was measured by placing the sensor unit in another cylindrical cell $\left(\varphi 40 \times 60 \mathrm{~mm}^{2}\right.$, inlet with an inner diameter of $2 \mathrm{~mm}$ on one side, and the same outlet on the other side) and evacuating the cylindrical cell with a pump. The pressure difference was about $0.2 \mathrm{hPa}$ at a flow rate of about $3 \mathrm{~L} / \mathrm{min}$. We found that liquid can be detected by the sensor by drawing in the surrounding air at a given suction power, vaporizing the material. 


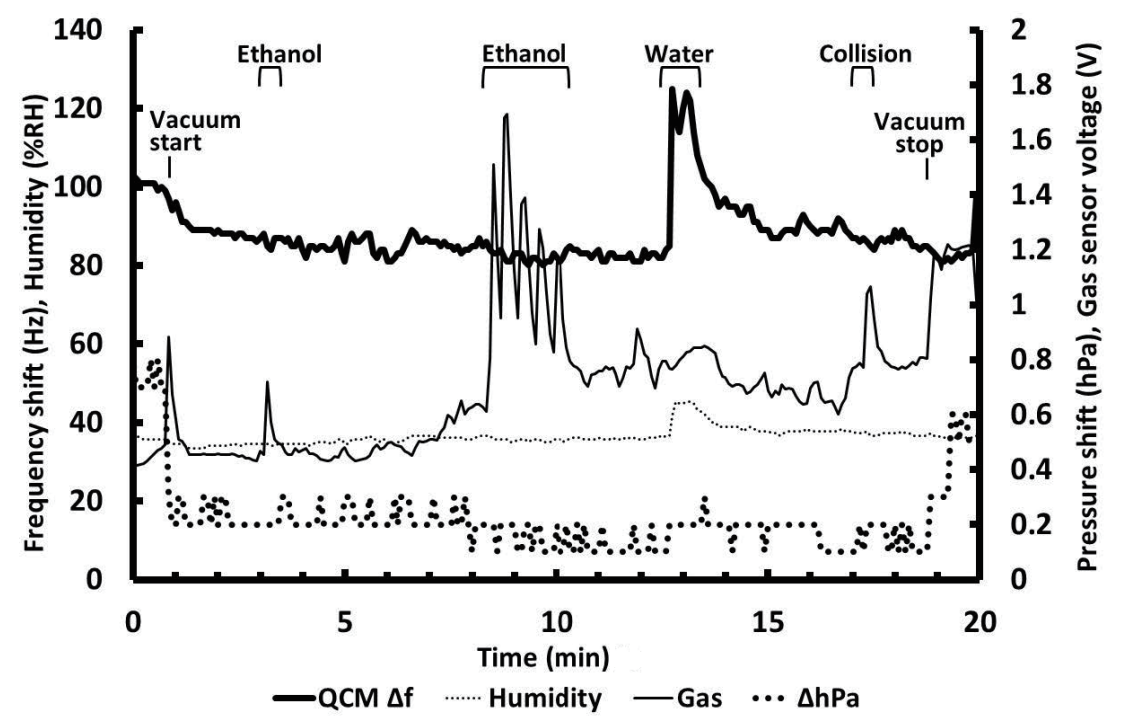

Fig. 4. Example of sensor unit data obtained using robot vacuum cleaner. The temperature was $25.4-25.8{ }^{\circ} \mathrm{C}$, the QCM was AT-cut, $f_{0}=12 \mathrm{MHz}$ with Ag electrodes, and the data recording interval of the sensor unit was $5 \mathrm{~s}$. The basic specifications of the Figaro TGS8100 semiconductor gas sensor can be seen at https://www.figaro.co.jp/ product/docs/tgs8100_product\%20information_jp_rev06.pdf (Last accessed 5 April 2019). ${ }^{*}$ Collision $=$ The robot vacuum cleaner collided with the tripod used for photography.

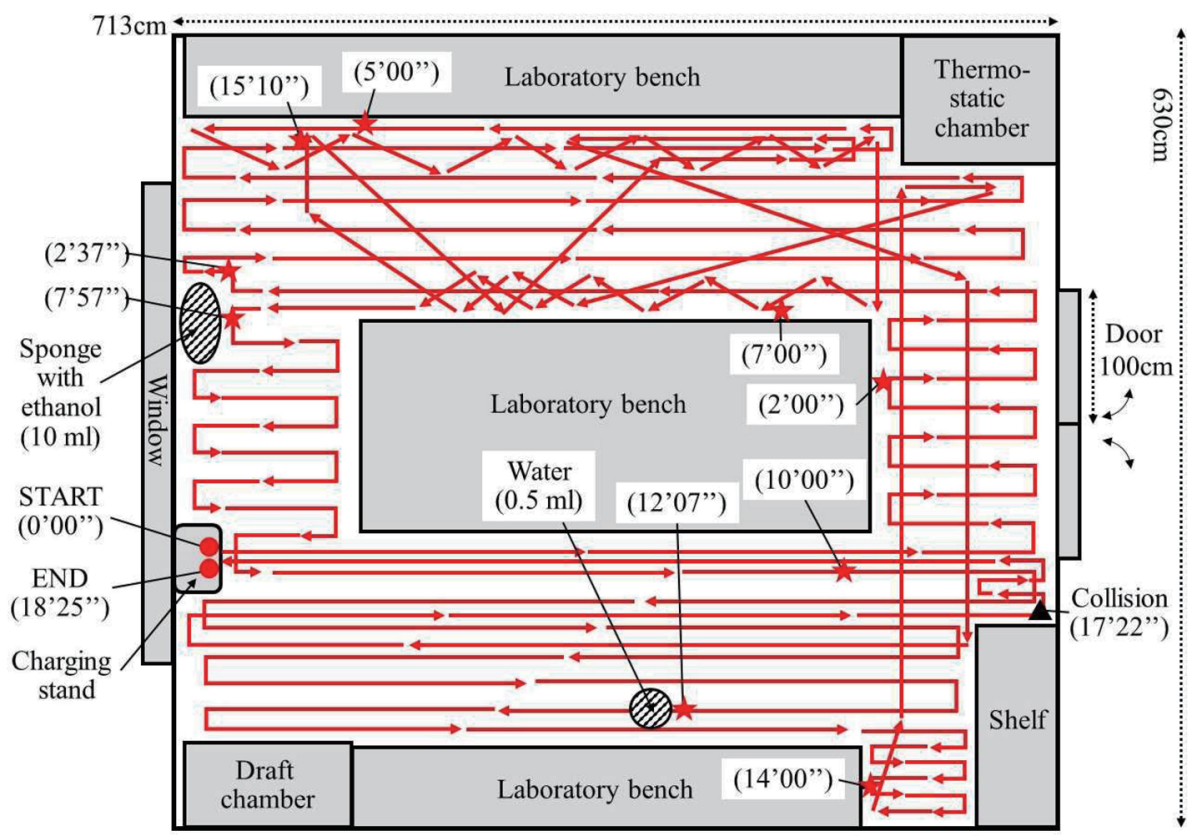

Fig. 5. (Color online) Example of the traveling route of the robot cleaner.

Next, for comparison, measurements were made using a conventional vacuum cleaner. The model (Panasonic; MC-PKL16A) used was of the ordinary paper-bag type, which is moved by hand. The conventional vacuum cleaner was moved in a manner that simulated the behavior of the robot vacuum cleaner as closely as possible. As with the robot vacuum cleaner, the sensor 
unit was placed in the paper dust bag. The suction power of the conventional vacuum cleaner in terms of the pressure difference between the air inlet and the air outlet was, according to the air pressure sensor output, about $40 \mathrm{hPa}$ at a high suction power, about $20 \mathrm{hPa}$ at a medium suction power, and about $10 \mathrm{hPa}$ at a low suction power. Figure 6 shows an example of the measurement data at a low suction power. The figure shows a pattern of detection characteristics similar to that for the robot vacuum cleaner. The semiconductor gas sensor outputs do not show a significant increase, with the changes in the outputs being comparable to those for the robot vacuum cleaner. Robot vacuum cleaners from many manufacturers have a rotating brush to transfer dust and particles to the dust box. The most likely reason for the increase in detection output is that a few water droplets on the floor were distributed by the brush and vaporized to inside the robot vacuum cleaner, where they were detected by the sensor unit in the dust box. In contrast, a conventional vacuum cleaner merely draws in air. Whether a spilled liquid is detected largely depends on the amount of leakage, the ambient air temperature, and the humidity. This indicates that the rotating brush assists in the detection of a spilled liquid on the floor.

Since the frequency of the QCM sensor changes with the mass of the substance adsorbed on the electrode, the frequency also changes with the adsorption of dust and particles. In this experiment, the sensor was covered with a filter to suppress these effects. Therefore, we consider that this measurement method was able to suppress the effects of dust and particles.

An indoor air conditioning unit was not used during the test. Because of this, the QCM sensor in particular produced some outputs that were most likely affected by outdoor weather conditions, such as the temperature and humidity. We need to perform an appropriate analysis using various data, including data on the inside of the cleaner and indoor environmental data.

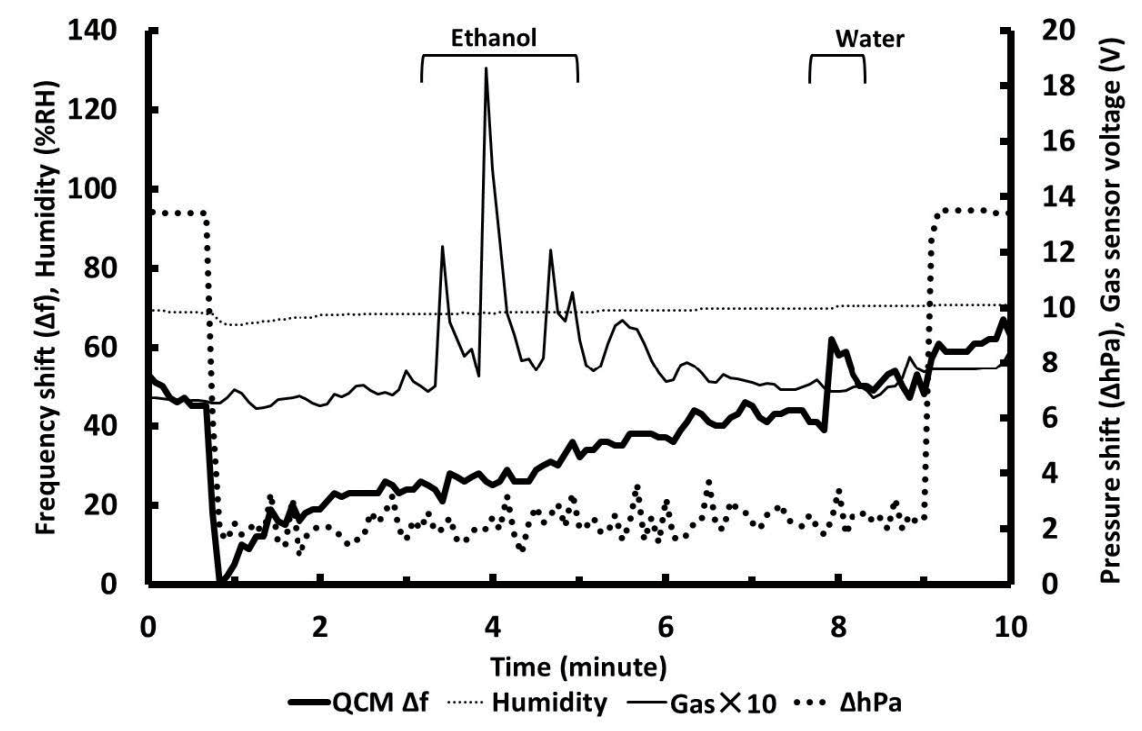

Fig. 6. Example of the sensor unit data obtained using ordinary paper-bag-type vacuum cleaner. The temperature was $22.3-24.1^{\circ} \mathrm{C}$, the QCM was AT-cut, $f_{0}=12 \mathrm{MHz}$ with Ag electrodes, and the data recording interval of the sensor unit was $5 \mathrm{~s}$. The basic specifications of the Figaro TGS8100 semiconductor gas sensor can be seen at https:// www.figaro.co.jp/product/docs/tgs8100_product\%20information_jp_rev06.pdf(Last accessed 5 April 2019). 


\section{Conclusions}

We investigated the effectiveness of passive indoor environmental measurement using a robot vacuum cleaner and showed that this form of monitoring has the potential to detect a spilled or leaked liquid. Robot vacuum cleaners are produced by several manufacturers. We showed that this measurement system can be applied by downsizing temperature, humidity, pressure, and gas sensors, and integrating them into a sensor unit that is mounted on a robot vacuum cleaner. To use this system for real-world applications, we need to collect information on the way that individual users use their robot vacuum cleaners and on the indoor environment, and analyze such information. Currently, robot vacuum cleaners are passive devices. However, we believe that, in the near future, they will be able to be actively guided by integrating AI technology with a wireless control system, such as Bluetooth or Wi-Fi.

\section{Acknowledgments}

This research was supported by a grant from the LIXIL JS Foundation.

\section{References}

1 J. W. Gardner and P. N. Bartlett: Electronic Noses: Principles and Applications (Oxford University Press, Oxford, 2000).

2 T. C. Pearce, S. S. Schiffman, H. T. Nagle, and J. W. Gardner: Handbook of Machine Olfaction: Electronic Nose Technology (Wiley-VCH, Weinheim, 2002).

3 Y. Sasaki, K. Hayashi, and K. Toko: Sens. Mater. 21 (2009) 191.

4 G. Ferri, M. V. Jakuba, A. Mondini, V. Mattoli, B. Mazzolai, D. R. Yoerger, and P. Dario: Rob. Auton. Syst. 59 (2011) 988.

5 H. Matsuo, Y. Furusawa, M. Imanishi, S. Uchida, and K. Hayashi: J. Rob. Mechatron. 24 (2012) 47.

6 Z. Chen and J. Wang: IEEE Sens. J. 17 (2017) 5963.

7 H. Ishida, K. Suetsugu, T. Nakamoto, and T. Moriizumi: Sens. Actuators A Phys. 45 (1994) 153.

8 L. Marques, U. Nunes, and A. T. de Almeida: Thin Solid Films 418 (2002) 51.

9 A. T. Hayes, A. Martinoli, and R. M. Goodman: Robotica 21 (2003) 427.

10 H. Ishida, G. Nakayama, T. Nakamoto, and T. Moriizumi: IEEE Sens. J. 5 (2005) 537.

11 W. Li, J. A. Farrell, S. Pang, and R. M. Arrieta: IEEE Trans. Rob. 22 (2006) 292.

12 T. Nakatsuka, Y. Kagawa, H. Ishida, and S. Toyama: Proc. IEEE Sensors 2006 (IEEE, 2006) 416.

13 A. J. Lilienthal, A. Loutfi, and T. Duckett: Sensors 6 (2006) 1616.

14 M. Trincavelli, M. Reggente, S. Coradeschi, A. Loutfi, H. Ishida, and A. J. Lilienthal: Proc. IEEE/RSJ International Conference on Intelligent Robots and Systems (IROS, 2008) 2210.

15 G. Kowadlo and R. A. Russell: Int. J. Rob. Res. 27 (2008) 869.

16 A. Loutfi, S. Coradeschi, A. J. Lilienthal, and J. Gonzalez: Robotica 27 (2009) 311.

17 K. Song, Q. Liu, and Q. Wang: Sensors 11 (2011) 2129.

18 J. Gonzalez-Jimenez, J. G. Monroy, and J. L. Blanco: Sensors 11 (2011) 6145.

19 J. Li, Q. Meng, Y. Wang, and M. Zeng: Auton. Rob. 30 (2011) 281.

20 H. Ishida, Y. Wada, and H. Matsukura: IEEE Sens. J. 12 (2012) 3163.

21 A. B. Rodríguez, A. R. G. Ramirez, E. R. De Pieri, A. L. Lopez, and A. D. C. de Albornoz: J. Med. Biol. Eng. 32 (2012) 453.

22 N. Ando, S. Emoto, and R. Kanzaki: Bioinspiration Biomimetics 8 (2013) 016008.

23 Y. Chen, H. Cai, Z. Chen, and Q. Feng: Build. Environ. 118 (2017) 101. 


\begin{abstract}
About the Authors
Kazutoshi Noda is a senior researcher at the National Institute of Advanced Industrial Science and Technology (AIST). He is interested in environmental measurement using a quartz crystal microbalance and gas sensing.

Hidenobu Aizawa is a senior researcher at the National Institute of Advanced Industrial Science and Technology (AIST). He is interested in biosensors and chemical sensors using a quartz crystal microbalance.
\end{abstract}

